\title{
Vimentin downregulation is an inherent feature of murine erythropoiesis and
} occurs independently of lineage

\author{
FRANK SANGIORGI, CATHERINE M. WOODS and ELIAS LAZARIDES \\ Division of Biology, 156-29, California Institute of Technology, Pasadena, CA 91125, USA
}

\begin{abstract}
Summary
In mammalian erythropoiesis, the mature cells of the primitive lineage remain nucleated while those of the definitive lineage are anuclear. One of the molecular and structural changes that precedes enucleation in cells of the definitive lineage is the cessation in the expression of the gene for the intermediate filament (IF) protein vimentin and the removal of all vimentin filaments from the cytoplasm. We show here that in immature primitive cells vimentin is synthesized and forms a cytoplasmic network of IFs. As differentiation proceeds in vivo, vimentin gene expression is downregulated in these cells; this is accompanied by the loss of vimentin filaments from the cytoplasm. This loss temporally coincides with
\end{abstract}

the nucleus becoming freely mobile within the cytoplasm, suggesting that, while IF removal is not directly linked to the physical process of enucleation, it may be a prerequisite for the initiation of nuclear mobility in both lineages. These changes are also observed in early primitive cells cultured in vitro, suggesting that they constitute an intrinsic part of the murine erythroid differentiation program independent of lineage and hematopoietic microenvironment.

Key words: murine erythropoiesis, intermediate filaments, primitive erythrocytes, vimentin, mouse.

\section{Introduction}

During vertebrate development, erythropoiesis occurs in a succession of distinct loci, with the sequential appearance of two erythroid lineages each possessing specific morphological and physiological characteristics (Sabin, 1920; Kovach et al. 1967; Bruns and Ingram, 1973). In both avian and mammalian species, the first lineage to be produced, the primitive lineage, originates in the blood islands of the yolk sac. The cells of this lineage are large (12-13 $\mu \mathrm{m}$ in diameter), amoeboid and nucleated, expressing characteristic embryonic hemoglobins. These cells differentiate as a cohort, being released into the circulation while still mitotically active (around 9 days of embryonic development for the mouse). They are short-lived, being progressively replaced by the definitive lineage of erythroid cells (from 12 to 13 days in the mouse) (Bruns and Ingram, 1973; Fantoni et al. 1967, 1969a).

The second lineage, the definitive lineage, arises in specific interim sites before the bone marrow becomes the ultimate erythropoietic site just prior to hatching or birth (Sabin, 1920; Bruns and Ingram, 1973; DieterlenLièvre and Martin, 1981; Russell and Bernstein, 1966; Fantoni et al. 1967, 1969b). In mammals, the first wave of definitive erythropoiesis occurs specifically in the fetal liver (Fantoni et al. 1969a,b). In mice the first hematoblasts can be distinguished at 10 days of gestation, developing within the liver before being released into the circulation as intermediate-sized $(8 \mu \mathrm{m}$ diameter) erythrocytes but with identical anuclear, biconvex, disc-shaped morphology to the smaller $(6 \mu \mathrm{m})$ spleen- and bone-marrow-derived erythrocytes found in circulation after birth (Russell and Bernstein, 1966; Fantoni et al. 1967, 1969a,b). These mammalian definitive erythrocytes differ markedly from their nonmammalian counterparts which remain nucleated and exhibit a biconcave disc morphology (Bruns and Ingram, 1973; Lazarides, 1987). Thus, despite the similar differentiation pathway from early BFU-e and CFU-e precursor to erythroblast cell stages (Till and McCulloch, 1980; Samarut and Gazzolo, 1982), mammals have an additional differentiation stage where the nucleus is actively extruded to give rise to an anuclear reticulocyte. This reticulocyte then matures into the characteristic biconcave disc-shaped erythrocyte.

Morphological studies have shown that enucleation is a complex process that involves an asymmetric positioning of the nucleus and a concentration of the erythroid membrane skeleton in the incipient reticulocyte prior to enucleation, a process resembling asymmetric cytokinesis (Geidushek and Singer, 1979). This step occurs only within the liver, spleen and bone marrow, and appears to involve the association of the nucleated 
erythroblasts with stromal cells, possibly a distinct subset of macrophages (Crocker et al. 1988). The extruded nuclei are then phagocytosed by the supportive macrophages. The exact role of cell-cell contact or extracellular factors in this morphogenetic process remains to be determined since murine erythroblasts transformed in vivo with the Friend murine leukemia viral complex will reliably differentiate and enucleate in vitro in the absence of supportive stromal cells or extracellular matrix (Koury et al. 1982, 1984). However, it is clear that, for enucleation to occur, multiple morphogenetic changes must have taken place during the divergence of nucleated and anuclear red blood cells.

One such change occurs in the structure of the cytoplasm that allows the nucleus to rotate and move freely, a prerequisite for its asymmetric positioning in the cytoplasm prior to extrusion. Normally, the nucleus is anchored to the plasma membrane and maintained in an invariant position. In avian red blood cells this has been shown to be effected by a network of vimentin intermediate filaments (IFs) (Granger and Lazarides, 1982; Virtanen et al. 1979). This vimentin network is augmented during the final stages of avian erythroid terminal differentiation in both primitive and definitive lineages resulting from the upregulation of vimentin expression at the transcriptional and translational levels (Blikstadt and Lazarides, 1983; Capetanaki et al. 1983). In contrast, in murine erythroleukemia (MEL) cells, used as a model of murine definitive erythroid differentiation, a marked and rapid downregulation in vimentin expression takes place when they are chemically induced to differentiate, with complete disappearance of vimentin IFs within $96 \mathrm{~h}$ (Ngai et al. 1984). Similar removal of vimentin filaments occurs in human erythroblasts grown in vitro (Dellagi et al. 1983). This divergence in vimentin expression appears to be due to changes in regulatory sequences in the chicken and mammalian vimentin genes and is effected at the late CFU-e stage (Ngai et al. 1987; Koury et al. 1989). These observations have led to the hypothesis that the removal of the vimentin IF network is a necessary early prerequisite for enucleation to proceed and one of the fundamental changes that has occurred in the divergence of the morphogenetic pathways of definitive avian and mammalian erythroid cells (Ngai et al. 1987).

In this report, we analyze the expression of vimentin during the differentiation of murine primitive erythrocytes and compare it with that in hepatic and adult definitive erythrocytes. We demonstrate that a vimentin IF network exists in primitive cells early in development. However, as differentiation proceeds, vimentin expression is markedly downregulated and is accompanied by the loss of the vimentin IF network. These changes occur at the same developmental stage when the primitive erythroblasts are cultured in vitro. The loss of IFs correlates with the nucleus becoming freely mobile within the cell. These results are consistent with the hypothesis that the downregulation and loss of vimentin IFs represent an intrinsic part of the erythroid differentiation program in both the primitive and definitive lineages and that these events are independent of both hematopoietic microenvironment and the physical process of enucleation.

\section{Materials and methods}

\section{Animals}

All mice were of the outbred MTS strain, bred and maintained at Harlan Sprague Dawley Laboratories (San Diego, CA). The dating of fetal development was based on timed matings of both hormonally primed and non-primed females, with day 0 of gestation commencing on the morning after mating.

\section{Cells}

Primitive erythroid cells were isolated by a modification of the procedure described by Kovach et al. (1967). At selected gestational ages ( 8 to 14 days), pregnant mice were sacrificed by cervical dislocation and their uteri removed and placed in calcium-/magnesium-free phosphate-buffered saline (PBS) containing $35 \mathrm{U} \mathrm{ml}^{-1}$ heparin. The embryos were then dissected out either within the decidua in the case of 8 and 9 day embryos or within the amniotic sac in the case of older embryos. After removal of extraembryonic tissues, erythroid cells were released by mechanical disruption of the yolk sac blood islands ( 8 to 10 days) or by transection of the dorsal aorta or umbilical vessels (older embryos). From 13 days of gestation onward, circulating cells within the embryo also included hepatic erythrocytes. The older the embryo, the cleaner the dissection from maternal tissues, including the placenta, with greatly reduced contamination by maternal erythrocytes. The cells were collected in Dulbecco's modified Eagle's medium (DMEM) with high glucose supplemented with $1 \%$ bovine serum albumin (BSA) and $35 \mathrm{Uml}^{-1}$ heparin. Residual tissue fragments and contaminating maternal leucocytes were removed by filtering the cell suspension through a combined cheese cloth-Leuko-Pak filter (Fenwal Laboratories, IL). The cells were subsequently collected by centrifugation and resuspended in DMEM. The cell suspension was further enriched for primitive or hepatic erythroid cells by centrifugation through a discontinuous step gradient of Percoll (Pharmacia LKB Biotechnology, NJ) in $0.15 \mathrm{M} \mathrm{NaCl}$. The gradient consisted of five $2 \mathrm{ml}$ steps of increasing Percoll densities ranging from 1.065 to $1.10 \mathrm{~g} \mathrm{ml}^{-1}$, the exact composition being modified for each time point to compensate for the increasing density of the primitive erythrocytes with gestational age due to accumulation of hemoglobin and their shrinking size. Typically, the primitive cells sedimented in the 1.075 to $1.08 \mathrm{~g} \mathrm{~m}^{-1}$ fraction at 9 days of gestation but by 14 days they cosedimented with mature definitive cells in the $1.1 \mathrm{~g} \mathrm{ml}^{-1}$ fraction. Hepatic erythroid cells were typically found in the 1.09 to $1.1 \mathrm{~g} \mathrm{ml}^{-1}$ fraction at 14 days of gestation. The cells were layered onto the gradient and centrifuged at $10000 \mathrm{revs} \mathrm{min}^{-1}$ for $10 \mathrm{~min}$ at $4^{\circ} \mathrm{C}$ in an SS34 rotor. $2 \mathrm{ml}$ fractions of the gradient were collected, diluted in DMEM and cells collected by centrifugation. Those fractions containing primitive or hepatic erythroid cells were identified by microscopy during cell counts. This identification was subsequently confirmed by nuclear staining (Paul, 1975) and benzidine staining for hemoglobin (Friedman and Schildkraut, 1977). Contamination of the primitive cell fraction with embryo-derived myeloid cells was insignificant as myelopoiesis does not commence until the 15 th or 16th day of gestation (Russell, 1979).

Culture of isolated yolk sac cells was carried out exactly as 
described by Cudennec et al. (1981). Murine fibroblasts were isolated from the ear shell of an MTS mouse and maintained in DMEM supplemented with $10 \%$ fetal calf serum (FCS), $40 \mathrm{U} \mathrm{ml}^{-1}$ penicillin and $40 \mu \mathrm{g} \mathrm{ml}^{-1}$ streptomycin. Avian erythrocytes were isolated from 15 day embryos as described by Blikstadt and Lazarides (1983).

\section{Electrophoresis and immunoblotting}

Cells were lysed in sodium dodecyl sulfate (SDS) sample buffer, and $1 \times 10^{6}$ cells were electrophoresed on a $7.5 \%$ polyacrylamide-SDS gel and transferred to nitrocellulose as previously described (Granger and Lazarides, 1984). After blocking at $37^{\circ} \mathrm{C}$ in Tris-buffered saline (TBS) containing $0.25 \%$ gelatin and $0.1 \%$ Tween-20 (TBS-GT) the filters were incubated overnight at room temperature in TBS-GT with a $1 / 1000$ dilution of either a rabbit anti-chicken vimentin (Granger and Lazarides, 1979) or a goat anti-mouse vimentin (ICN, Costa Mesa, CA) antiserum. After washing, the filters were incubated in TBS-GT containing ${ }^{125}$ I-Protein A for $30 \mathrm{~min}$ at room temperature, washéd extensively, dried and exposed to Kodak XAR- 5 X-ray film at $-80^{\circ} \mathrm{C}$. Alternatively, immunoblotting was performed on two-dimensional gels. For this the cells were either lysed directly in $9 \mathrm{M}$ urea or fractionated into Triton X-100 soluble and insoluble fractions as described by Blikstadt and Lazarides (1983). The insoluble fraction was resuspended in Triton-saline lysis buffer $(1 \%$ Triton X-100, $150 \mathrm{~mm} \mathrm{NaCl}, 10 \mathrm{~mm}$ Tris- $\mathrm{HCl}, \mathrm{pH} 7.5,5 \mathrm{~mm}$ $\mathrm{MgCl}_{2}, 2 \mathrm{~mm}$ EGTA, $6 \mathrm{~mm} \beta$-mercaptoethanol, $1 \mathrm{~mm}$ phenylmethylsulphonyl fluoride, $50 \mathrm{U} \mathrm{ml}^{-1}$ aprotinin), sonicated with two $15 \mathrm{~s}$ pulses at 100 watts and centrifuged for $5 \mathrm{~min}$ at 12000 revs min $^{-1}$ in an Eppendorf centrifuge to give a cytoskeletal supernatant fraction free of nuclei. The soluble and cytoskeletal fractions were then denatured in $9 \mathrm{M}$ urea, $5 \mathrm{~mm}$ $\beta$-mercaptoethanol. Two-dimensional electrophoresis was performed by a modification of the method of O'Farrell (1975) as described by Hubbard and Lazarides (1979), using a $12.5 \%$ polyacrylamide-SDS gel in the second dimension. For the immunoblotting of two-dimensional gels, the vimentin antiserum was visualized using the Protoblot alkaline phophatase-conjugated goat anti-rabbit IgG system (Promega Corp., Madison, WI)

\section{Metabolic labelling and autoradiography}

Primitive erythrocytes $\left(6 \times 10^{6}\right)$ from 10 - to 14-day embryos were washed with methionine-free minimum essential medium (MEM) supplemented with 10\% dialysed FCS and incubated in $0.5 \mathrm{ml}$ of this medium for $15 \mathrm{~min}$ prior to addition of $400 \mu \mathrm{Ci}\left[{ }^{35} \mathrm{~S}\right]$ methionine $\left(1100\right.$ to $1200 \mathrm{Ci} \mathrm{mmol}^{-1}$; Amersham Corp., IL). After $1 \mathrm{~h}$ at $37^{\circ} \mathrm{C}$ the cells were washed with DMEM and lysed in Triton-saline lysis solution. Cytoskeletal and soluble fractions were prepared as described above, denatured in $9 \mathrm{~m}$ urea, $6 \mathrm{~mm} \beta$-mercaptoethanol and subjected to two-dimensional electrophoresis. The resultant gel was processed for autoradiography by impregnating with 2,5diphenyloxazole (PPO), dried and exposed to XAR-5 X-ray film as previously described (Ngai et al. 1987). Incorporation of $\left[{ }^{35} \mathrm{~S}\right]$ methionine into protein in total lysates or fractionated samples was determined as trichloroacetic acid (TCA)-precipitable counts.

\section{Immunofluorescence}

Vimentin filaments in murine primitive cells were visualized by immunofluorescence essentially as described by Granger and Lazarides (1982), with the following modifications. Primitive red cells from 9 to 13 days of gestation or cultured primitive cells were allowed to settle onto coverslips at room temperature. Adherent cells were fixed in $2 \%$ formaldehyde in TBS for 2 min, rinsed and permeabilized in TBS containing $1 \%$ Triton X-100. Polyclonal anti-chicken vimentin (Granger and Lazarides, 1979), anti-chick protein 4.1 (Granger and Lazarides, 1984), anti-chicken $\beta$-spectrin (Nelson and Lazarides, 1983) or anti-mouse tubulin (kindly provided by Dr J. Olmsted) all at $1 / 40$ dilution were added to the coverslips. Cells were subsequently incubated in fluorescein isothiocyanate-conjugated goat anti-rabbit IgG diluted 1/150 (MilesYeda Ltd., Rehovot, Israel). Coverslips were mounted in Elvanol and viewed with a Leitz phase-epifluorescence microscope using a $63 \times$ lens.

\section{$R$ Nase protection analysis}

Steady-state mRNA levels of murine vimentin and $\gamma$-actin were assayed by protection from RNase digestion of ${ }^{32} \mathrm{P}$ labelled RNA probes synthesized in vitro (Melton et al. 1984; Zinn et al. 1983). For detection of vimentin sequences a $315 \mathrm{bp}$ $E c o$ RI-HincII fragment from a $1.4 \mathrm{~kb}$ murine vimentin cDNA was subcloned into the pGEM-4 vector (Promega Co., Madison, WI). This fragment is derived from a cDNA isolated from a mouse spleen library and corresponds to the sequence towards the $5^{\prime}$ end of vimentin mRNA extending from nucleotides 324 to 630 (Wood et al. 1989). After linearizing the recombinant plasmid with $P v u \amalg$, a 383 nucleotide ${ }^{32} \mathrm{P}$-labelled RNA probe was generated with SP6 polymerase as described (Ngai et al. 1987). Murine $\gamma$-actin mRNA was detected with a 145 nucleotide ${ }^{32} \mathrm{P}$-labelled RNA probe synthesized from a Hinfl linearized template containing the $3^{\prime}$ non-coding region of the human $\gamma$-actin cDNA (Gunning et al. 1983) cloned into pSP64 (Enoch et al. 1986), as previously described (Ngai et al. 1987).

Cell lysates were hybridized with probe using the protocols of Firestein et al. (1987) and Thompson and Gillespie (1987) with the following modifications. Primitive erythroid cells isolated from 9 to 14 days of gestation and cultured mouse fibroblasts were washed with DMEM, counted and pelleted. The cell pellets were dissolved in $4 \mathrm{M}$ guanidinium thiocyanate, $25 \mathrm{~mm}$ sodium citrate, $\mathrm{pH} 7.0,100 \mathrm{~mm} \beta$-mercaptoethanol to give a suspension of $10^{8}$ cells $/ \mathrm{ml}$. Aliquots of the lysates were stored at $-70^{\circ} \mathrm{C}$ until used.

The hybridization mixture typically contained 1 to $2 \times 10^{5} \mathrm{cts} \mathrm{min}^{-1}$, corresponding to 1 to $2 \mathrm{ng}$ of lyophilized probe and lysate equivalent to 1 to $2 \times 10^{6}$ primitive erythroid cells or $5 \times 10^{4}$ fibroblasts in a final volume of $25 \mu \mathrm{l}$ in $4 \mathrm{M}$ guanidinium thiocyanate, $25 \mathrm{~mm}$ sodium citrate, $\mathrm{pH} 7.0$, $100 \mathrm{~mm} \beta$-mercaptoethanol. The mixture was heated to $65^{\circ} \mathrm{C}$ for $5 \mathrm{~min}$ and incubated at $22^{\circ} \mathrm{C}$ for $17 \mathrm{~h}$. The hybridization reaction was diluted with $500 \mu \mathrm{l}$ of $10 \mathrm{~mm}$ Tris- $\mathrm{HCl}, \mathrm{pH} 7.5$, $5 \mathrm{~mm}$ EDTA, $0.3 \mathrm{M} \mathrm{NaCl}, 900 \mathrm{U} \mathrm{ml}^{-1}$ RNase $\mathrm{T} 1$ and $500 \mu \mathrm{g} \mathrm{ml}^{-1}$ RNase A. After $1 \mathrm{~h}$ at $30^{\circ} \mathrm{C}$, SDS and proteinase $\mathrm{K}$ were added to give a final concentration of $0.6 \%$ and $0.3 \mathrm{mg} \mathrm{ml}^{-1}$, respectively, and the incubation continued at $37^{\circ} \mathrm{C}$ for $30 \mathrm{~min}$. The digests were extracted with phenolchloroform-isoamyl alcohol and coprecipitated with $10 \mu \mathrm{g}$ of yeast tRNA in isopropanol and lyophilized. The pellets were resuspended in $90 \%$ formamide, $1 \mathrm{~mm}$ EDTA, $0.1 \%(\mathrm{w} / \mathrm{v})$ xylene cyanole and the fragments were resolved on a $5 \%$ polyacrylamide- $8 \mathrm{~m}$ urea gel. The gel was exposed to Kodak XAR-5 film with an intensifying screen. Protection of the in vitro synthesized vimentin probe by cellular vimentin mRNA should yield a 307 nucleotide fragment while protection of the $\gamma$-actin probe should yield an 80 nucleotide fragment.

\section{Flow cytometry}

Acridine orange (AO) cell cycle analysis as developed by Darzynkiewicz et al. $(1980 \mathrm{~b})$, was performed on 10 to 14 day primitive cells that had been previously fixed and stored in 
$50 \%$ ethanol at $4^{\circ} \mathrm{C}$. Cells were resuspended in physiologica saline at a concentration of $10^{6} \mathrm{ml}^{-1}$ immediately before use. To an aliquot of $2 \times 10^{5}$ cells, $0.4 \mathrm{ml}$ of $80 \mathrm{mM} \mathrm{HCl}, 0.2 \%$ Triton X-100 in saline was added. After $30 \mathrm{~s}$ of permeation the cells were stained by adding $1.2 \mathrm{ml}$ of $126 \mathrm{~mm} \mathrm{NaHPO}, 37 \mathrm{~mm}$ acetic acid, $1 \mathrm{~mm}$ EDTA, $150 \mathrm{~mm} \mathrm{NaCl}$ and $10 \mu \mathrm{g} \mathrm{ml}^{-1}$ of AO. To confirm the specificity of AO staining for RNA, 10 day primitive cells were permeabilized and treated with $1000 \mathrm{U} \mathrm{ml}^{-1}$ of RNase $A$ in $150 \mathrm{~mm}$ sucrose, $5 \mathrm{mM} \mathrm{MgCl}$, $20 \mathrm{~mm}$ Tris- $\mathrm{HCl}, \mathrm{pH} 6.5$ at $37^{\circ} \mathrm{C}$ for $30 \mathrm{~min}$ prior to staining with $\mathrm{AO}$. Samples were filtered through a $50 \mu \mathrm{m}$ mesh prior to fluorescent-activated cell sorting (FACS) analysis. Analysis of AO-stained samples was performed on an Ortho Cytofluorograph $50 \mathrm{H}$ using an argon laser excitation beam of $250 \mathrm{~mW}$ at $488 \mathrm{~nm}$ as described (Boyer et al. 1989).

\section{Results}

\section{Vimentin IFs expressed in early murine primitive} erythroblasts are lost during differentiation

To establish whether murine nucleated primitive erythroblasts express a vimentin-based IF network at any stage during their development, purified primitive cells from 9 to 14 days of gestation were fixed and processed for immunofluorescence using a polyclonal anti-chicken vimentin antiserum which is known to react with both avian and murine vimentin with high affinity (Granger and Lazarides, 1979; Ngai et al. 1987). In primitive red cells from 9 and 10 days of gestation this antiserum detects a distinct IF network extending from the nucleus to the cell membrane (Fig. 1A and B). However, as these cells mature, they shrink in size and the network becomes reduced exhibiting a diffuse distribution between day 11 and 12 until by day 13 it has disappeared completely from the cell (Fig. 1C to E). One striking correlation with this loss of vimentin IFs was readily seen by microscopy when living cells were flushed under the coverslip. From 13 days onwards, the nuclei of the primitive cells were completely mobile, tumbling within the cell as they floated across the field of view. Hepatic erythrocytes from the circulation of 13 or 14 day embryos, readily distinguishable from their bone-marrow-derived counterparts on the basis of size ( $8 \mu \mathrm{m}$ compared to $6 \mu \mathrm{m}$ in diameter), were completely negative for vimentin.

For comparison, immunofluorescence was performed on these primitive cells using anti- $\beta$-spectrin, protein 4.1 and tubulin antibodies. Spectrin and protein 4.1 are components of the erythroid membrane skeleton and would be expected to accumulate under the membrane during terminal differentiation. Tubulin is the main structural component of the characteristic marginal band found encircling the equator of nucleated definitive cells in nonmammalian vertebrates (GoniakowskaWitalinska and Witalinski, 1976). It was therefore of interest to determine whether these structural domains were also present in primitive murine red cells. A diffuse uniform fluorescence, localized under the plasma membrane, was observed with anti- $\beta$-spectrin and protein 4.1 antibodies. This fluorescence became more intense as these cells matured (data not shown). Immunofluorescence with anti-tubulin revealed the presence of a loosely organized band of microtubules around the cell periphery in non-mitotic cells around 10 to 12 days as reported by Koury et al. (1987). However, these microtubules subsequently vanished from the cytoplasm from 13 days of gestation onwards (data not shown)

To confirm that the putative IF network was indeed vimentin, immunoblot analysis was performed on samples prepared from erythroid cells isolated between 8 and 14 days of gestation. It should be noted that with 8 day embryos it was difficult to obtain primitive cells completely free of the contaminating maternal erythrocytes. For this time point the loading was based on the number of primitive cells in the sample. Since adult erythrocytes do not express vimentin, any signal must arise solely from the primitive cells. As seen in Fig. 2, the anti-chicken vimentin antibody readily detects a single band of $M_{\mathrm{r}} 57000$ of equal intensity on a per cell basis in primitive cells from 8 and 9 day embryos, which comigrates with the band detected in murine lymphocytes, cells known to express vimentin (Lilienbaum et al. 1986; Dellagi et al. 1983), and has a slightly slower electrophoretic mobility than chicken vimentin (Granger and Lazarides, 1979). The signal then rapidly declines from 10 days onwards, such that after day 12 it can no longer be detected even after overexposure of the immunoblot (Fig. 2, lower panel). Since hepatic erythrocytes are released into the circulation around 12 to 13 days of gestation and can be separated readily from the primitive cell population, these cells were also analyzed for the presence of vimentin. As seen in Fig. 2, fetal hepatic red cells are also negative for vimentin. Immunoblotting of two-dimensional gels of 10 day primitive cells confirmed that this immunoreactive protein comigrates as murine vimentin (Fig. 3A and B), migrating adjacent to $\alpha$ - and $\beta$-tubulins.

\section{Cessation of vimentin expression during primitive erythroblast differentiation}

To assess the relative changes in vimentin synthesis during primitive erythroid development, cells were pulse labeled with $\left[{ }^{35} \mathrm{~S}\right]$ methionine and the amount of vimentin synthesized and assembled was monitored by two-dimensional gel electrophoresis of both the soluble and cytoskeletal cell fractions followed by autoradiography. Newly synthesized vimentin can readily be visualized by such analysis (Blikstadt and Lazarides, 1983; Ngai et al. 1987). Fig. 3 depicts the area of the autoradiographs that include vimentin, the tubulins and actin in the cytoskeletal fractions. Newly synthesized vimentin was not evident in the soluble fraction (data not shown), consistent with the previous observation that any vimentin synthesized is rapidly assembled into filaments (Blikstadt and Lazarides, 1983). Newly synthesized, cytoskeletal vimentin is readily visualized in autoradiograms of 10 day primitive cell cytoskeletal fractions but the amount of vimentin synthesized rapidly declines thereafter needing much longer exposure times to be visualized for 11 and 12 day cells, being undetectable in 13 and 14 day cells (Fig. 3B and 

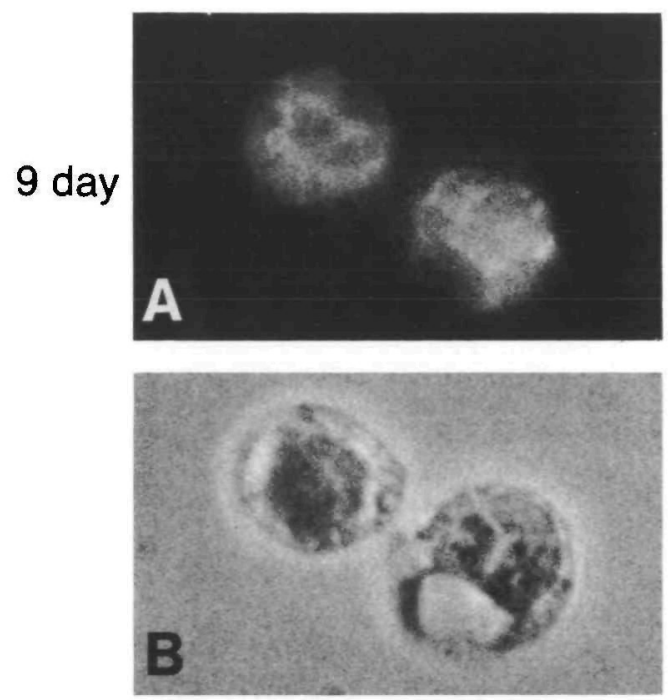

11 day
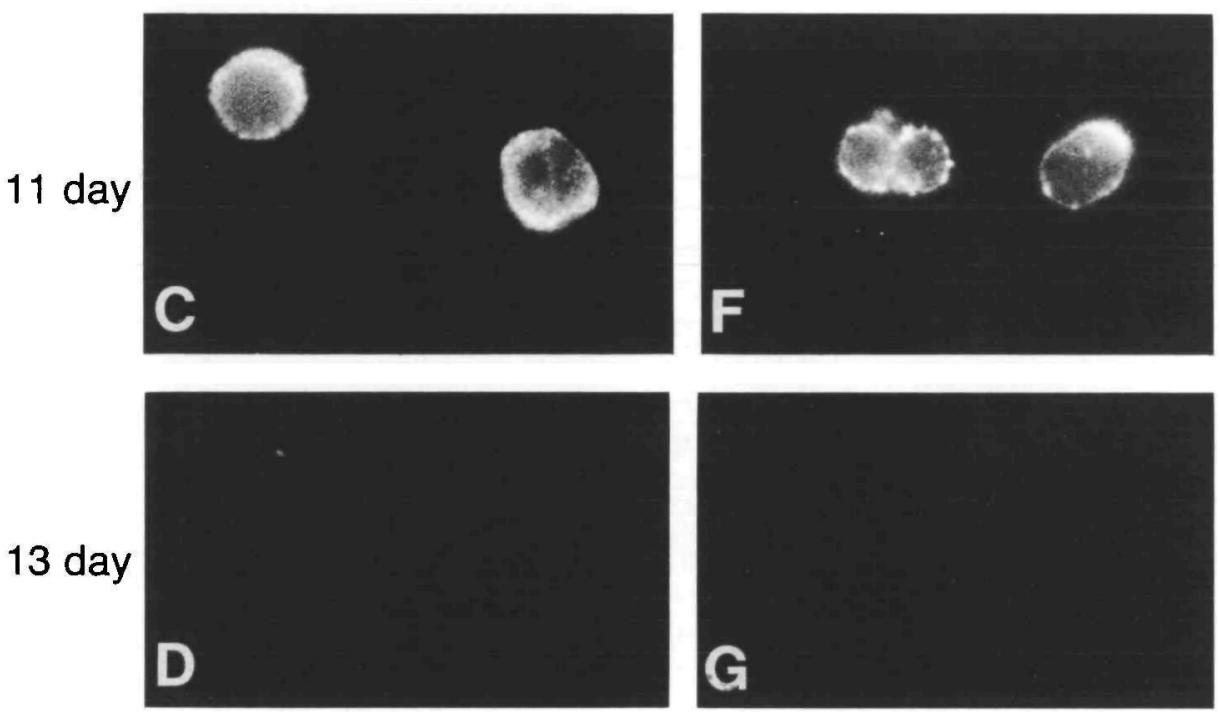

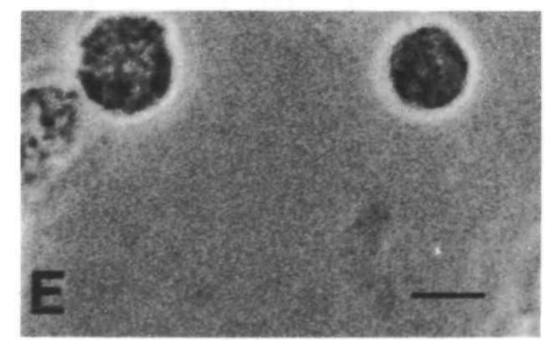

in vivo

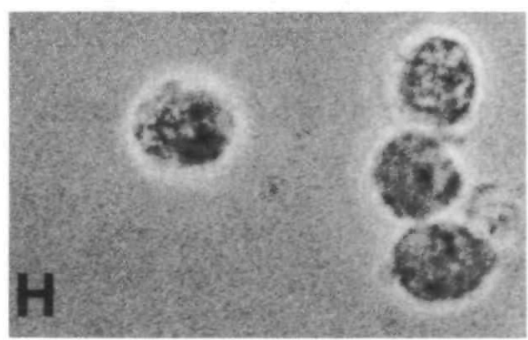

in vitro
Fig. 1. Immunofluorescence localization of vimentin during murine erythropoiesis. Murine primitive erythroblasts were isolated from embryos at $9(\mathrm{~A}, \mathrm{~B})$, $11(C)$ and $13(D, E)$ days of gestation, left panels (in vivo); or isolated from 9 day embryos and grown for $2(\mathrm{~F})$ and $4(\mathrm{G}, \mathrm{H})$ days in culture (i.e. they correspond to 11 and 13 days of in vivo development, respectively), right panels (in vitro). Cells were processed for immunofluorescence using a vimentin-specific antibody and viewed with epifluorescence $(A, C, D, F, G)$ or phase contrast (B,E,H) optics. Note the loss of vimentin from day 11 to 13 whether grown in vivo or cultured in vitro. Bar, $10 \mu \mathrm{m}$.
C). This decline is much more precipitous than the overall decline in total protein synthesis occurring during the terminal differentiation of these cells as determined by the incorporation of $\left[{ }^{35} \mathrm{~S}\right]$ methionine into TCA-precipitable counts (data not shown). RNase protection analysis of RNA was then performed in order to correlate these changes in protein synthesis with changes in mRNA levels. As seen in Fig. 4, a decline in vimentin mRNA levels exactly correlates with changes in synthesis of the protein. As illustrated in Fig. 4A, a 307 nucleotide fragment is protected by cellular RNA from 9 day cells but not by RNA from 12 , 13 or 14 day cells. By using twice the lysate $\left(2 \times 10^{6}\right.$ cell equivalents) vimentin mRNA can be reliably detected in 10 day cells but appears to be absent from 12 day cells (Fig. 4B).

Among other cytoskeletal proteins readily identified in the two-dimensional gel autoradiograms, the synthesis of tubulins also declines precipitously around day 12 (Fig. 3B and C), an event similar to the downregulation seen during MEL cell-induced differentiation (Ngai et al. 1987). This correlates with the loss of peripheral microtubules seen by immunofluorescence described above. In contrast, actin synthesis declines in parallel with the general decline in protein synthesis, with newly synthesized actin being readily visible up to 


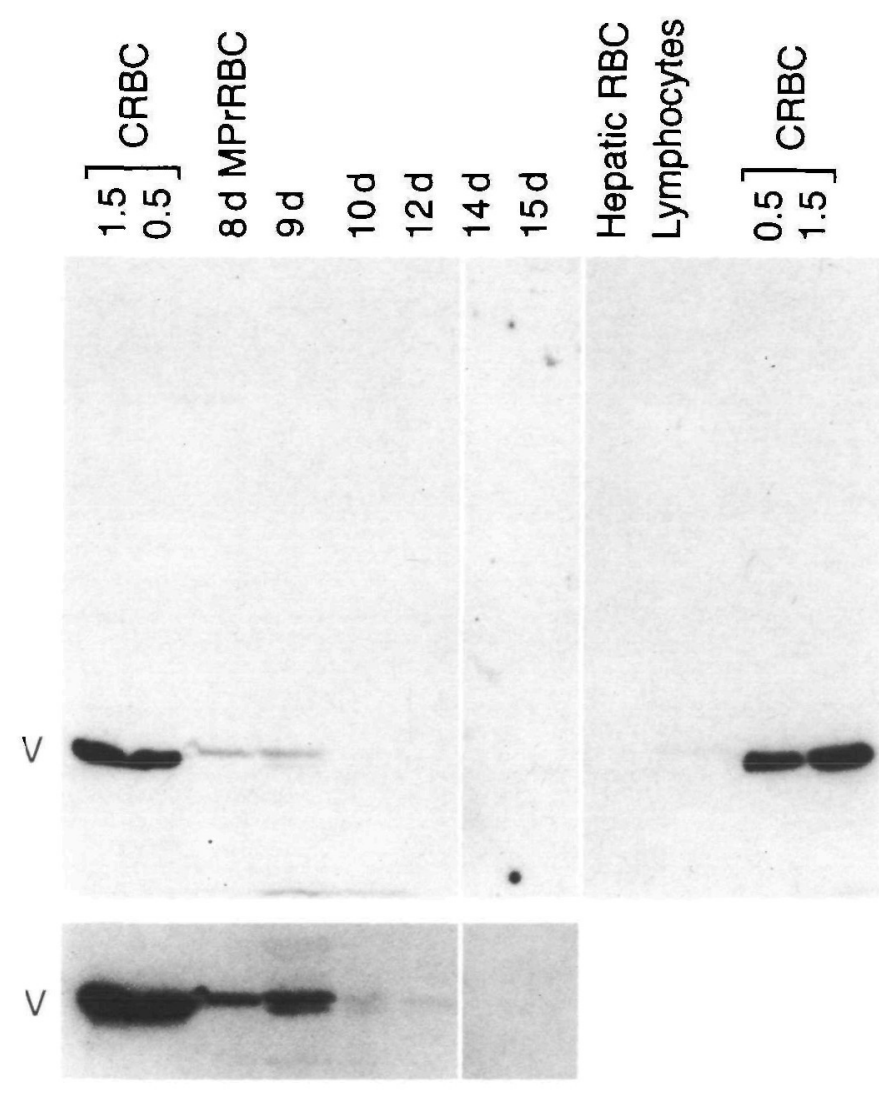

Fig. 2. Steady-state levels of vimentin during primitive erythroid cell differentiation. Total cell lysates from definitive chicken erythrocytes, murine primitive red cells and murine lymphocytes were solubilized in SDS buffer and subjected to immunoblot analysis. CRBC 1.5 and 0.5 refer to loadings of a chicken erythrocyte lysate equivalent to 1.5 and $0.5 \times 10^{6}$ cells, respectively. Murine primitive cells (MPrRBC) from 8, 9, 10, 12, 14 and 15 days were $1.5 \times 10^{6}$ cell equivalents per lane. Definitive hepatic erythroid cells (isolated from embryonic circulation on day 14 of gestation) and lymphocytes (purified from adult mouse blood) were both $1.5 \times 10^{6}$ cell equivalents. The upper, larger panel represents an exposure of $3 \mathrm{~h}$ with intensifying screen. The lower panel presents a 3-day exposure without screen of the corresponding lanes immediately above. $V$ indicates vimentin. Note the difference in $M_{\mathrm{r}}$ between avian and mouse vimentin with the gel system employed here (Ngai et al. 1987).

14 days (Fig. 3B and C). Furthermore, the steady-state level of $\gamma$-actin mRNA (Fig. 4C) parallels the continued synthesis of actin through the later days of differentiation (Fig. 3B and C).

\section{Downregulation of the vimentin IF network in primitive cells grown in culture}

To determine whether this pattern of vimentin regulation in murine primitive cells is an intrinsic property of these cells' differentiation program or is due to external factors provided by the yolk sac, purified 9 day primitive cells were grown in culture for up to 4 days and the presence of a vimentin network was then assayed by immunofluorescence after different times of culture.
We chose to use 9 day cells for this experiment because of the difficulty of obtaining sufficient numbers of purified 8 day cells. Given that the steady-state level of vimentin was constant up to the 9 day stage, it seemed reasonable to suppose that those events leading to vimentin downregulation had not yet occurred. As shown in Fig. 1F a diffuse vimentin network was evident for up to two days in culture, but after 4 days of culture this network had disappeared (Fig. $1 \mathrm{G}$ and $\mathrm{H}$ ). Immunoblot analysis confirmed that this disappearance of vimentin filaments correlated with the loss of vimentin protein from these cells (data not shown). Hence, the disappearance of the vimentin network follows the same time course in vitro as in in vivo.

\section{Changes in mitotic state and RNA content of primitive} erythrocytes during differentiation

FACS cell cycle analysis of primitive erythrocytes isolated on different days of gestation was performed to examine how the pattern of vimentin downregulation compares with changes in the mitotic state during differentiation. The cytogram (RNA versus DNA content) of 10 day primitive cells (Fig. 5) is characteristic of a proliferating cell population and is comparable to that of an MEL cell population in exponential growth (Darzynkiewicz et al. 1980a; Traganos et al. 1979). From the eleventh day onward, however, the RNA/DNA profile of this population changes. The relative content of RNA decreases in conjunction with a shift in DNA content from $4 \mathrm{C}$ to $2 \mathrm{C}$ (where $\mathrm{C}$ is the DNA content per haploid nucleus) so as to compress the cytogram pattern towards the $\mathrm{G}_{\mathrm{o}}$ compartment of the cell cycle. The twelfth day therefore represents the point when primitive cells begin to cease proliferation and become quiescent such that by day 13 and 14 the vast majority of cells are quiescent. The withdrawal of these cells from the cell cycle and the appearance of the terminally differentiated state would tentatively place these cells in the $\mathrm{G} 1_{\mathrm{D}}$ compartment (Darzynkiewicz, 1987). However, the altered accessibility of chromosomal DNA to small intercalating agents such as ethidium bromide or $\mathrm{AO}$, and the degree to which the chromatin is condensed, both characteristic of $\mathrm{G} 1_{D}$ cells (Darzynkiewicz et al. 1980), remain to be determined for these primitive cells.

Treatment of 10 day cells with RNase $A$ at $37^{\circ} \mathrm{C}$ for $30 \mathrm{~min}$ abolishes the $\mathrm{y}$-axis component of the cytogram while leaving the histogram of DNA content intact (Fig. 5). This confirms the specificity of AO staining for RNA and reveals the contribution of RNA to the pattern of the cytogram. By comparing the RNA data from the cytograms in Fig. 5, 10 day cells are seen to have a broad range of RNA content. As these cells mature, progressively more cells exhibit a lower RNA content with this shift in relative values becoming quite marked by 12 days of gestation and being most pronounced by day 13 .

\section{Discussion}

Enucleation is a process unique to mammalian defini- 

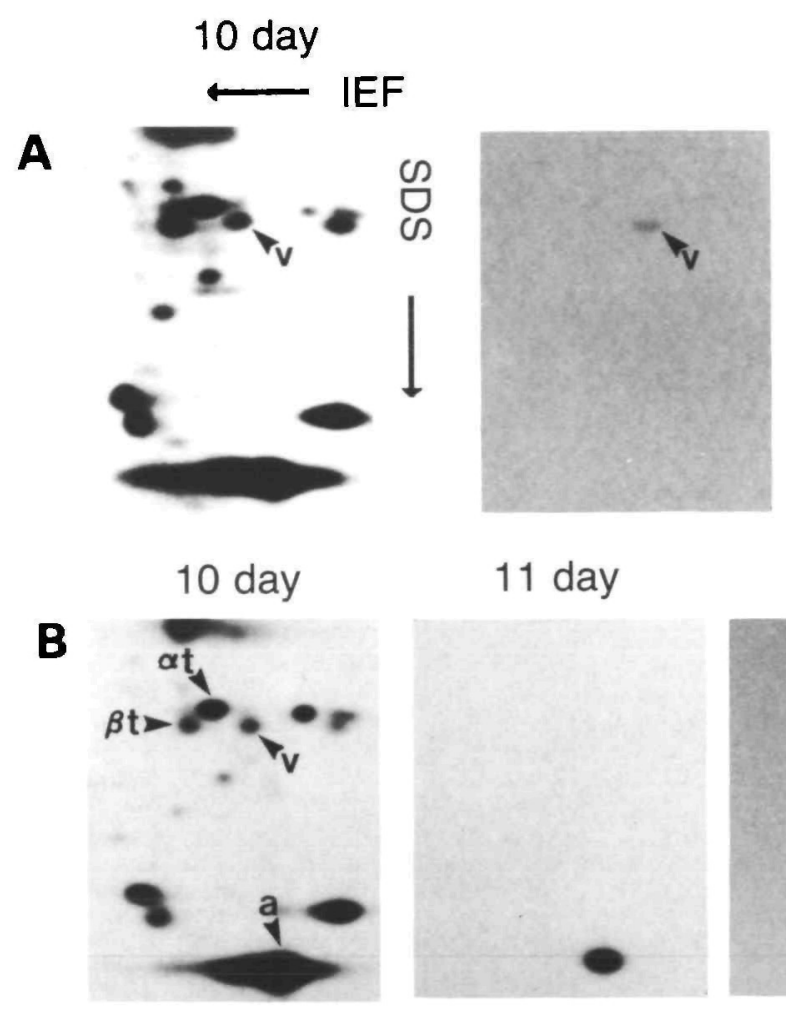

12 day

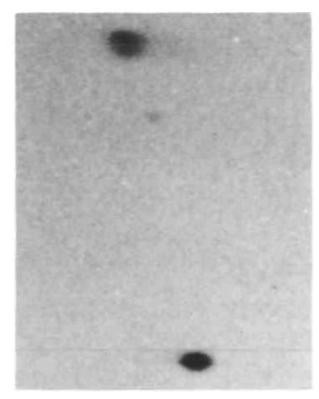

C
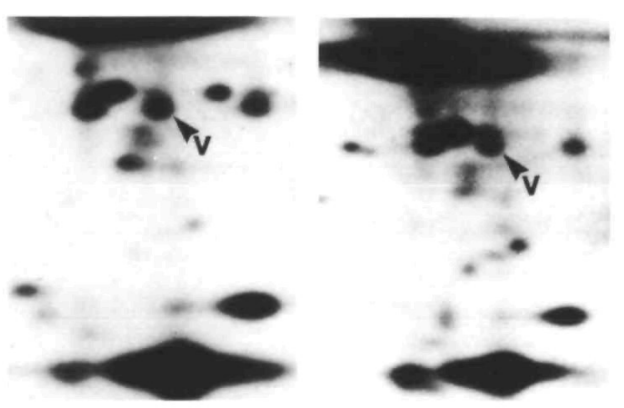

13 day
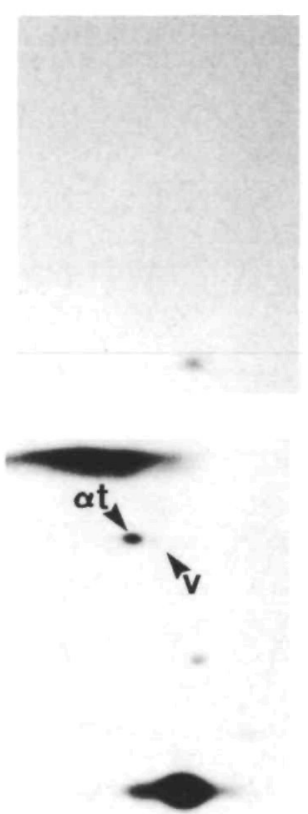

14 day
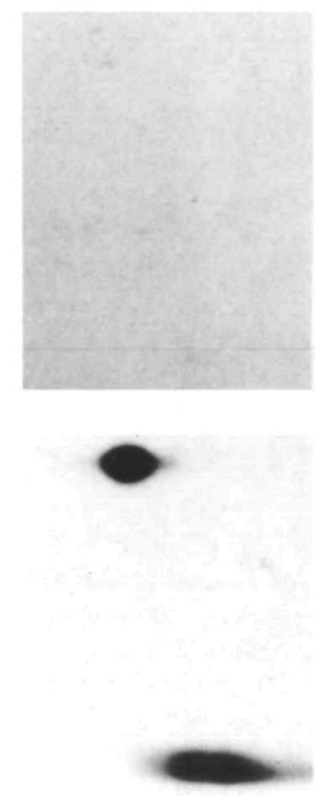

Fig. 3. Relative synthetic rate of vimentin, tubulins and actins during primitive erythroid cell differentiation. All panels represent two-dimensional electrophoretic patterns of $\left[{ }^{35} \mathrm{~S}\right]$ methionine-labelled cytoskeletal fraction of primitive red cells isolated at the designated days of gestation. Only a small area of the total two-dimensional gel, corresponding to the region containing tubulin, actin and vimentin, is shown. (A) Autoradiograph of a Western blot of the cytoskeletal fraction of $3 \times 10^{6}$ 10 day primitive cells labelled for $1 \mathrm{~h}$ with $\left[{ }^{35} \mathrm{~S}\right]$ methionine (left panel) and the same filter incubated with an anti-vimentin antibody and developed with alkaline phosphatase to detect vimentin (right panel). Exposure of the autoradiogram was for 7 days. (B) Fluorograms of two-dimensional gels of cytoskeletal fractions equivalent to $10^{6}$ primitive cells labeled at different days of development. Fluorograms were exposed for 2 days. Symbols: $\alpha t, \alpha$-tubulin; $\beta \mathrm{t}, \beta$-tubulin; a, actin; $v$, vimentin.

(C) Extended exposure ( 3 weeks) of the same 11 to 14 day gels presented immediately above. Note the loss of vimentin by day 12 .

tive erythropoiesis. Since erythrocytes of the primitive lineage remain nucleated, a comparative analysis of the definitive and primitive lineages should delineate those changes that have occurred independently of the physical process of enucleation and those molecular changes that are lineage specific and intimately coupled to this process. In nonmammalian nucleated erythrocytes, two major structural domains exist that are absent in anuclear mammalian definitive red cells. The first is a marginal band of microtubules that forms around the equatorial plane of the cell periphery during terminal differentiation (Behnke, 1970; Barrett and Dawson,
1974). The second is the vimentin-based IF network that emanates from the nucleus and anchors it to the plasma membrane at a distinct and separate site from the marginal band attachment sites (Granger and Lazarides, 1982; Virtanen et al. 1979). Given the apparent structural linkage of the IF network and the nucleus, the hypothesis has been advanced that the dissolution of this network is a necessary prerequisite for enucleation to proceed (Ngai et al. 1987). A comparison of vimentin expression in avian definitive and murine primitive and definitive erythropoiesis therefore provides an opportunity to examine how the expression 


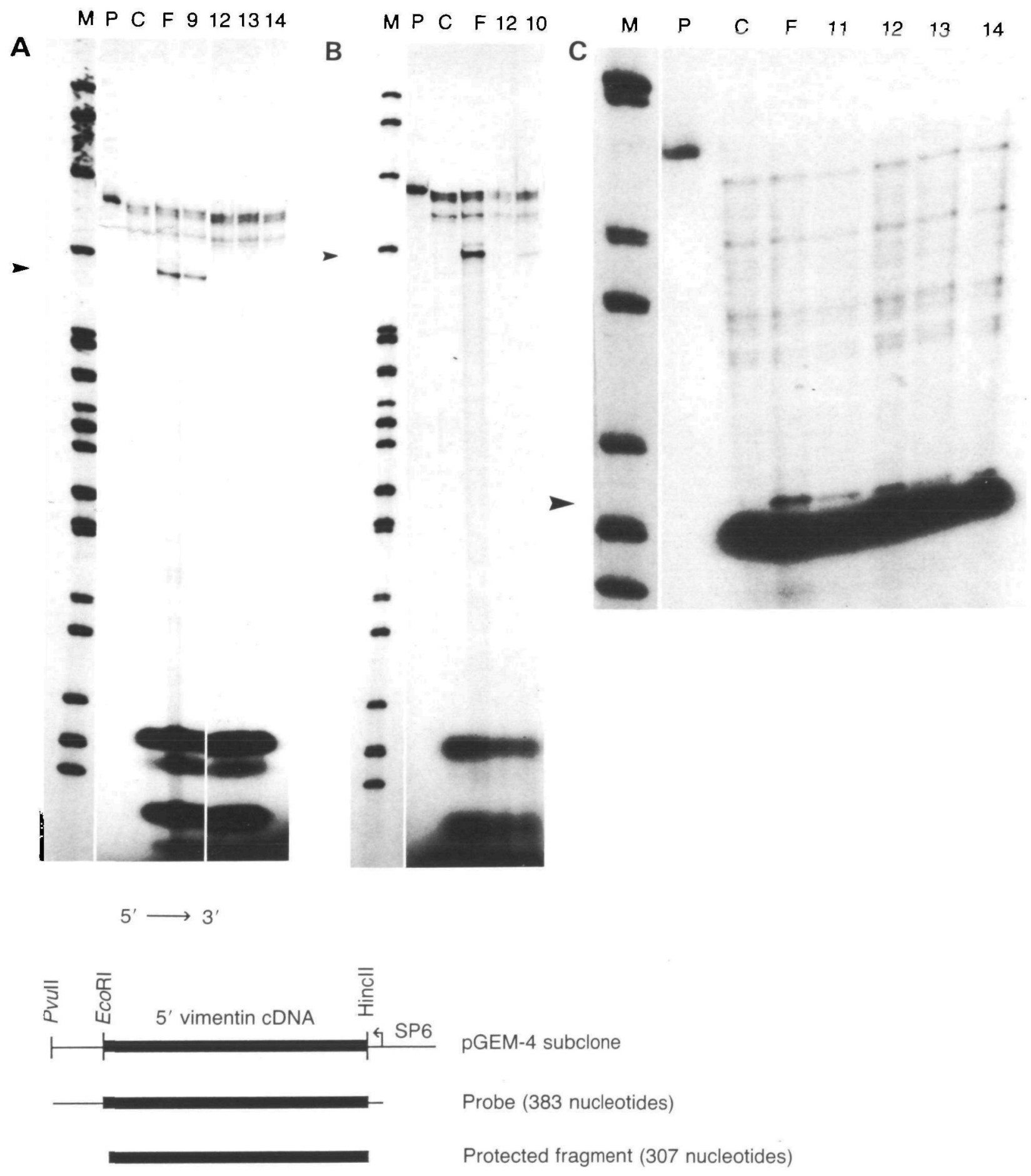

of this gene relates to the process of enucleation. We have shown here that murine primitive cells contain an IF network early in their development which is similar to that found in avian erythroblasts and early mammalian definitive erythroblasts. However, during the subsequent stages of terminal differentiation vimentin expression is downregulated and the IF network is removed as occurs during terminal differentiation of the murine definitive lineage (Ngai et al. 1984, 1987). Other workers have analyzed the cytoskeleton of 12 day murine primitive erythroid cells, demonstrating the presence of an IF network and peripheral microtubules, and concluded that murine primitive cells resemble nonmammalian definitive erythrocytes (Koury et al. 1987). As we have shown here such a resemblance is only transitory, with both the IF network and peripheral microtubules being removed during the final stages of differentiation. Thus, the downregulation of vimentin expression and the subsequent removal of IFs has occurred in both murine lineages irrespective of whether or not the nucleus is retained. However, we have observed that the initiation of filament removal in 
Fig. 4. RNase protection assays of vimentin and $\gamma$-actin mRNAs. Steady-state levels of vimentin and $\gamma$-actin mRNAs were determined as described in Materials and methods. Panels A and B represent two separate experiments. (A) Lane M, ${ }^{32} \mathrm{P}$-end-labeled pBR322 $\mathrm{Hpa \Pi}$ fragments as markers; lane $P$, probe in the absence of RNases; lane $C$, control digestion of probe in the presence of RNases and $10 \mu \mathrm{g}$ of yeast tRNA; lane F, probe protection by $5 \times 10^{4}$ cell equivalents of murine fibroblast lysate; lanes 9 to 14 , probe protection by $1 \times 10^{6}$ cell equivalents of 9 to 14 day primitive cells. The arrowhead indicates the expected 307 nucleotide fragment protected by vimentin mRNA. Autoradiographic exposure for 5 days.

(B) Lane $F$, protection of probe with $5 \times 10^{4}$ cell equivalents of fibroblast lysate; lane 12 , protection with $2 \times 10^{6}$ cell equivalents of 12 day primitive red cells; lane 10 , protection with $2 \times 10^{6}$ cell equivalents of 10 day primitive cells. Arrowhead indicates the expected 307 nucleotide fragment from vimentin mRNA. Exposure time, 7 days. The diagram beneath $A$ and $B$ indicates the pGEM-4 subclone containing the 315 base pair (bp) murine vimentin cDNA insert, the RNA probe of 383 nucleotides generated from that subclone, and the 307 nucleotide fragment specifically protected by vimentin mRNA. (C) Enlarged region of autoradiogram illustrating the RNase protection assay for $\gamma$-actin mRNA. Lane M, 147, 122, 110, 90, 76 and $67 \mathrm{bp}$ subset of pBR322 HpaП markers; lane P, the 145 nucleotide probe generated from the $\gamma$-actin subclone in the absence of nucleases; lanes 11 to 14, probe protection by $1 \times 10^{6}$ cell equivalents of 11 to 14 day primitive cells. Arrowhead indicates the protected fragment of 80 nucleotides.

primitive erythroblasts coincides temporally with the nucleus becoming mobile within the cytoplasm. Prior to enucleation, the nucleus must become freely mobile within the cell to allow its polar apposition to the plasma membrane (Geidushek and Singer, 1979). The results presented here argue that while IF removal in itself is not sufficient for enucleation to occur, it may be necessary to allow nuclear rotation and asymmetric positioning to occur.

In both normal and transformed murine definitive erythroblasts, the downregulation of vimentin expression and the concomitant removal of cytoplasmic

Fig. 5. Cell cycle analysis of murine primitive erythrocytes at selected days of development. AO staining of primitive cell populations was carried out as described in Materials and methods. Each panel consists of both a histogram of DNA content (green fluorescence) $v s$. cell number and a two-color cytogram of RNA content (red fluorescence) $v s$. DNA content (green fluorescence). Typically 2C DNA content gives a mean fluorescence intensity of about 198 (arbitrary units) and 4C DNA content yields a mean fluorescence of about 430 in these analyses. Panel $10 \mathrm{~d}+\mathrm{RNase}$ represents 10 day primitive red cells treated with $1000 \mathrm{U} \mathrm{ml}^{-1}$ of RNaseA prior to AO staining. The lower left-hand box in each cytogram (minimum RNA, 2C DNA content) represents $G_{0}$ cells. The upper left box labeled ' 2 ' (increased RNA, 2C DNA content) includes $G_{1}$ cells. Cells in the middle box labeled ' 3 ' (DNA content between $2 \mathrm{C}$ and $4 \mathrm{C}$ ) are taken to approximate the S-phase population. Cells in the right-most box labelled ' 4 ' ( $4 \mathrm{C}$ DNA content) are taken to represent $\mathrm{G}_{2}+\mathrm{M}$ cells.
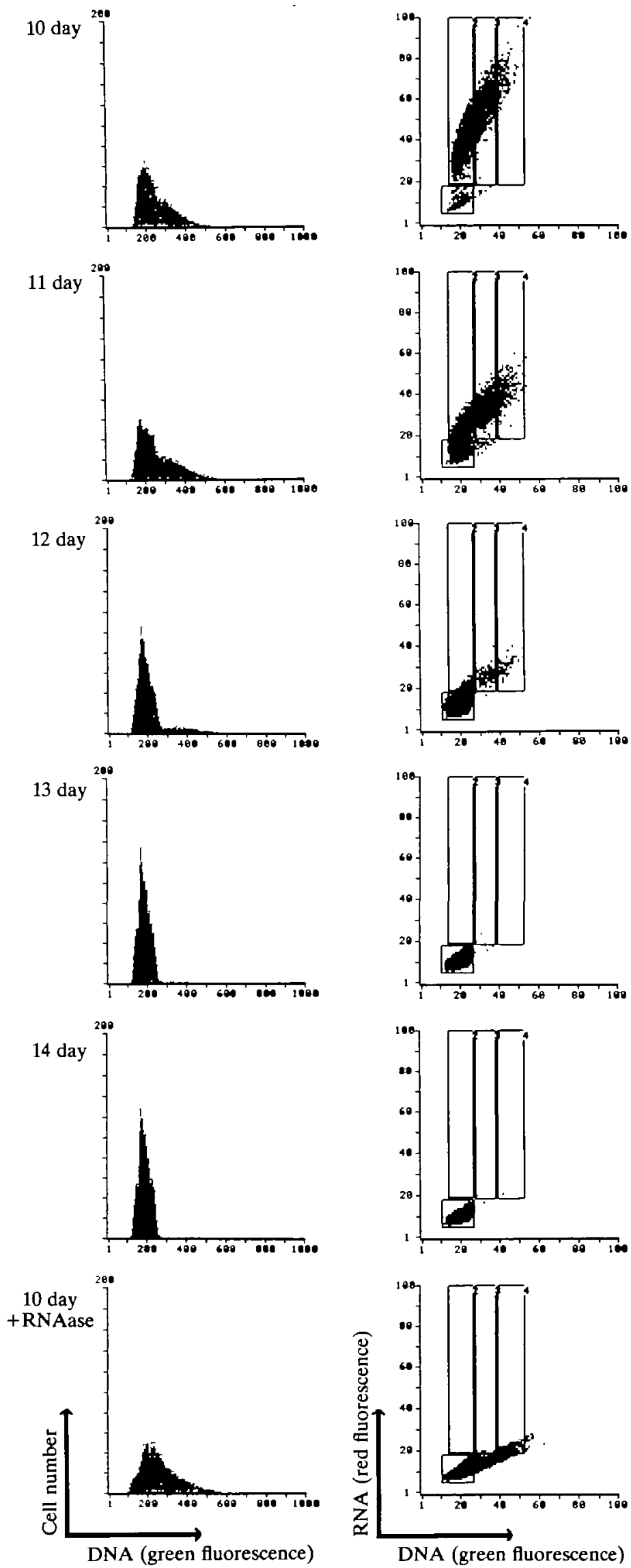
vimentin filaments occurs around the late CFU-e stage, since it is seen within $12 \mathrm{~h}$ of induced differentiation in MEL cells, before the onset of globin expression ( $\mathrm{Ngai}$ et al. 1984), and in early erythroblasts in vitro (Dellagi et al. 1983).

Although it remains to be determined whether the primitive lineage progresses through the same precursor cell stages as the definitive pathway, it is evident that the downregulation of vimentin expression is an early event in the terminal stages of primitive red cell differentiation. Hemoglobinized primitive cells can be first distinguished at 8 days of gestation within the blood islands. At 9 days of gestation they are released into the embryonic circulation where they differentiate synchronously becoming fully mature around day 14 to 15 (Kovach et al. 1967; Fantoni et al. 1968, 1969b; de la Chapelle et al. 1969). By following the expression and steady-state level of vimentin in primitive cells between 8 and 14 days of gestation, we have shown that a vimentin network exists in early primitive erythroblasts from day 8 to 10 . However, from day 10 onwards there is a dramatic decrease in the amount of vimentin mRNA and protein being synthesized accompanied by a loss of the vimentin IF network so that by day 13 vimentin expression has ceased and filaments are no longer detectable in these cells. These changes occur independently of continued association with the yolk sac microenvironment since identical changes occur over a similar time scale when 9 day cells are cultured in vitro either together with or independently of their yolk sac environment arguing that they must represent an intrinsic part of the primitive erythroid differentiation pathway.

FACS analysis of primitive cells from different days of gestation reveals that day 12 represents the stage of differentiation when murine primitive cells begin to exit from the mitotic cycle. This observation is in agreement with earlier conclusions based on thymidine labeling, that these cells cease DNA synthesis and hence become postmitotic around day 12 (de la Chapelle et al. 1969). Therefore, vimentin downregulation and filament removal commences just prior to the time the cells exit the mitotic cycle and enter their final phase of differentiation. By FACS analysis, the total RNA content of these primitive cells declines from day 11 onward; this is in accordance with other studies that have demonstrated a marked decline in total RNA and protein synthesis between day 11 and 15 (Kovach et al., 1967; Fantoni et al. 1968; de la Chapelle et al. 1969). Globin synthesis persists relatively unchanged until day 14 , presumably because of the relatively stable nature of globin mRNAs in these cells (Fantoni et al. 1968, $1969 a$ ). Since vimentin downregulation commences around day 10 , a stage at which the cells possess the DNA and RNA characteristics of an exponentially growing population, it must represent a specific repression event occurring before these general changes in RNA and protein synthesis. The precipitous decline in vimentin protein synthesis suggests that the vimentin mRNA is short-lived, but the mechanisms involved in the rapid removal of the pre-existing vimentin network remain to be determined. Dilution through cell division can not be the only contributing factor since cell division ceases by day 12 (de la Chapelle et al. 1969). Likewise, the activation of specific proteases at this stage is an unlikely explanation since chicken vimentin filaments can still accumulate while the endogenous murine vimentin is being eliminated in differentiating MEL cells harboring the chicken vimentin gene (Ngai et al. 1987). The observation that vimentin filaments are dynamic, undergoing rapid assembly and disassembly (Ngai et al. 1990) suggests that upon initiation of vimentin downregulation filaments begin to disassemble.

Assuming that this downregulation of vimentin is an important prerequisite for the process of enucleation, the question then arises why enucleation does not proceed in murine primitive erythroblasts following the release of mobility restraints on the nucleus. One possibility is that cells of the primitive lineage do not synthesize and/or assemble the appropriate receptors for polarized apposition of the nucleus to the plasma membrane and subsequent extrusion from the cytoplasm. Alternatively, the process of enucleation may require the direct interaction of these cells with a specific subset of hematopoietic stromal cells. It has been shown that erythroblasts in the process of enucleation within the bone marrow stroma are in close association with a discrete subset of macrophages which appear to be responsible for the phagocytosis of the extruded nuclei (Crocker et al. 1988). It is possible that the necessary supportive cells are not present in the yolk sac. Indeed, macrophage production is thought not to occur within the early yolk sac, but rather within the embryo around 14 days of murine embryogenesis (Russell, 1979). Irrespective of the reason why primitive cells do not enucleate, the results presented here and those of previous studies suggest that evolution of the cellular processes leading to enucleation has occurred in at least two stages. One is lineage independent and entails cytoplasmic structural changes that culminate in nuclear mobility. The other is the physical process of enucleation itself which is unique to the mammalian definitive lineage and may also be dependent on the stromal composition of the hematopoietic site.

We thank Rochelle Diamond for her expert assistance with FACS analysis, Jessica Dausman for help with mouse embryo manipulations, Drs Thomas Coleman and Michael Rodriguez for valuable comments on the manuscript, and Louise Cloutier and Dr Olivier Gandrillon for assistance with the figures. This work was supported by grants from the National Institutes of Health (AGO60 78A) and from the Lucille P. Markey Charitable Trust to the Division of Biology at the California Institute of Technology. F.S. was supported by fellowships from the Anna Fuller Fund and the American Heart Association (Greater Los Angeles Affiliate). C.W. was supported by a fellowship from the American Heart Association (Greater Los Angeles Affiliate) and by a grant from the American Cancer Society.

\section{References}

Barrett, L. A. And Dawson, R. B. (1974). Avian erythrocyte 
development: microtubules and the formation of the disc shape. Devl Biol. 36, 72-81.

BEHNKE, O. (1970). A comparative study of microtubules of discshaped blood cells. J. Ultrastruct. Res. 31, 61-75.

BlikstadT, I. AND LAZARIDES, E. (1983). Vimentin filaments are assembled from a soluble precursor in avian erythroid cells. $J$. Cell Biol. 96, 1803-1808.

Boyer, P. D., Diamond, R. A. And Rothenberg, E. V. (1989). Changes in inducibility of IL-2 receptor a-chain and T-cell receptor expression during thymocyte differentiation in the mouse. J. Immunol. 142, 4121-4130.

Bruns, G. A. P. and Ingram, V. M. (1973). The erythroid cells and hemoglobins of the chick embryo. Philos. Trans. R. Soc. London B. 266, 225-269.

Capetanaki, Y. G., NGai, J., Flytzanis, C. N. and Lazarides, E. (1983). Tissue specific expression of two mRNA species transcribed from a single vimentin gene. Cell 35, 411-420.

Crocker, P. R., Morris, L. AND Gordon, S. (1988). Novel cell surface adhesion receptors involved in the interaction between stromal macrophages and hematopoietic cells. J. Cell Sci. Suppl. 9, 185-206.

Cudennec, C. A., Thiery, J.-P. and Le Douarin, N. M. (1981)

In vitro induction of adult erythropoiesis in early mouse yolk sac. Proc. natn. Acad. Sci. USA 78, 2412-2416.

DARZYNKIEWICZ, Z. (1987). Cytochemical probes of cycling and quiescent cells applicable to flow cytometry. In Techniques in Cell Cycle Analysis (ed. J. W. Gray and Z. Darzynkiewicz). pp. 255-290. Humana Press, Clifton, NJ.

Darzynkiemicz, Z., Sharpless, T., Staiano-Coico, L. and Melamed, M. R. (1980b). Subcompartments of the $G_{1}$ phase of cell cycle detected by flow cytometry. Proc. natn. Acad. Sci. U.S.A. 77, 6696-6699.

Darzynkiewicz, Z., Traganos, F. and Melamed, M. R. (1980a). New cell cycle compartments identified by multiparameter flow cytometry. Cytometry 1, 98-108.

de la Chapelle, A., Fanton, A. and Marks, P. A. (1969). Differentiation of mammalian somatic cells: DNA and hemoglobin synthesis in fetal mouse yolk sac erythroid cells. Proc. natn. Acad. Sci. USA 63, 812-819.

Dellagi, K., Vainchenker, W., Vinci, G., Paulin, D. and Brouet, J. C. (1983). Alteration of vimentin intermediate filament expression during differentiation of human hemopoietic cells. EMBO J. 2, 1509-1514.

Dieterlen-Lièvre, F. and Martin, M. (1981). Diffuse intraembryonic hemopoiesis in normal and chımeric avian development. Devl Biol. 88, 180-191.

Enoch, T., Zinn, K. AND Maniatis, T. (1986). Activation of the human $\beta$-interferon gene requires an interferon-inducible factor. Mol. Cell Biol. 6, 801-810.

Fantoni, A., Bank, A. And Marks, P. A. (1967). Globin composition and synthesis of hemoglobins in developing fetal mice erythroid cells. Science 157, 1327-1329.

Fantoni, A., de la Chapelle, A., Chui, D., Rifkind, R. A. And MARKS, P. A. (1969b). Control mechanisms of the conversion of embryonic to adult hemoglobin. Annals New York Acad. Sci. 165, 194-204.

Fantoni, A., de la Chapelle, A. and Marks, P. A. (1969a). Synthesis of embryonic hemoglobins during erythroid cell development in fetal mice. J. biol. Chem. 244, 675-681.

Fanton, A., De la Chapelle, A., Rifkind, R. A. and Marks, P. A. (1968). Erythroid cell development in fetal mice: synthetic capacity for different proteins. J. molec. Biol. 33, 79-91.

Firestein, G. S., Gardner, S. M. and Roeder, W. D. (1987). Quantitative molecular hybridization with unfractionated, solubilized cells using RNA probes and polyacrylamide gel electrophoresis. Anal. Biochem. 167, 381-386.

Friedman, E. A. And Schildkraut, C. L. (1977). Terminal differentiation in cultured Friend erythroleukemia cells. Cell 12, 901-913

Geiduschek, J. B. And Singer, S. J. (1979). Molecular changes in the membranes of mouse erythroid cells accompanying differentiation. Cell 16, 149-163.

GoniakowSKa-WITALINSKa, L. AND WITALINSKI, W. (1976). Evidence for a correlation between the number of marginal band microtubules and the size of vertebrate erythrocytes. J. Cell Science 22, 397-401.

Granger, B. L. ANd Lazarides, E. (1979). Desmin and vimentin coexist at the periphery of the myofibril $\mathrm{Z}$ disc. Cell 18 , $1053-1063$

Granger, B. L. and Lazarides, E. (1982). Structural associations of synemin and vimentin filaments in avian erythrocytes revealed by immunoelectron microscopy. Cell 30, 263-275.

Granger, B. L. AND Lazarides, E. (1984). Membrane skeletal protein 4.1 of avian erythrocytes is composed of multiple variants that exhibit tissue specific expression. Cell 37, 595-607.

Gunning, P., Ponte, P., Okayama, J., Engel, J., Blau, H. and KEDES, L. (1983). Isolation and characterization of full-length cDNA clones for human alpha-, beta-, and gamma-actin mRNAs: skeletal but not cytoplasmic actins have an aminoterminal cysteine that is subsequently removed. Mol. cell. Biol 3, 787-795.

Hubbard, B. D. and Lazarides, E. (1979). Copurification of actin and desmin from chicken smooth muscle and their copolymenzation in vitro to intermediate filaments. J. Cell Biol. 80, $166-182$.

Koury, M. J., Bondurant, M. C., Duncan, D. T., Krantz, S. B. and Hankins, W. D. (1982). Specific differentiation events induced by erythropoietin in cells infected in vitro with the anemia strain of Friend virus. Proc. natn. Acad. Sci. USA 79 $635-639$.

Koury, M. J., Sawyer, S. T. and Bondurant, M. C. (1984). Splenic erythroblasts in anema-inducing Friend disease: a source of cells for studies of erythropoietin-mediated differentiation. $J$. cell. Physiol. 121, 526-532.

Koury, S. T., Koury, M. J. and Bondurant, M. C. (1989). Cytoskeletal distribution and function during the maturation and enucleation of mammalian erythroblasts. J. Cell Biol. 109, 3005-3013.

Koury, S. T., Repasky, E. A. And EckerT, B. S. (1987). The cytoskeleton of isolated murine primitive erythrocytes. Cell Tissue Res. 249, 69-77.

Kovach, J. S., Marks, P. A., Russell, E. S. and Epler, H. (1967). Erythroid cell development in fetal mice: ultrastructural characteristics and hemoglobin synthesis. J. molec. Biol. 25 , 131-142.

LAZARIDES, E. (1987). From genes to structural morphogenesis: the genesis and epigenesis of a red blood cell. Cell 51, 345-356.

Lilienbaum, A., Legagneux, V., Portier, M. M., Dellagi, K. AND PaUlin, D. (1986). Vimentın gene: expression in human lymphocytes and in Burkitt's lymphoma cells. $E M B O \mathrm{~J} .5$, 2809-2814.

Melton, D. A., Krieg, P. A., Rebagliati, M. R., Maniatis, T., ZiNN, K. AND GREEN, M. R. (1984). Efficient in vitro synthesis of biologically active RNA and RNA hybridization probes from plasmids containing bacteriophage SP6 promoter. Nucl. Acids Res. 12, 7035-7056.

Nelson, W. J. and Lazarides, E. (1983). Switching of subunit composition of muscle spectrin dunng myogenesis in vitro. Nature 304, 364-368.

NGal, J., Bond, V. C., Wold, B. J. and Lazarides, E. (1987). Expression of transfected vimentin genes in differentiating murine erythroleukemia cells reveals divergent cis-acting regulation of avian and mammalian vimentin sequences. Mol Cell Biol. 7, 3955-3970.

Ngai, J., Capetanaki, Y. G. and Lazarides, E. (1984).

Differentiation of murine erythroleukemia cells results in the rapid repression of vimentin gene expression. J. Cell Biol. 99 306-314.

Ngai, J., Coleman, T. and Lazarides, E. (1990). Localization of newly synthesized vimentin subunits reveals a novel mechanism of intermediate filament assembly. Cell 60, 415-427.

O'Farrell, P. H. (1975). High resolution two-dimensional electrophoresis of proteins. J. biol. Chem. 250, 4007-4021

Paul, J. (1975). Cell and Tissue Culture, 5th ed. p. 364. Churchill Livingstone, New York.

RusselL, E. S. (1979). Hereditary anemias of the mouse: a review for geneticists. Adv. Genet. 20, 357-459. 
Russell, E. S. AND Bernstern, S. E. (1966). Blood and blood formation. In The Biology of the Laboratory Mouse (ed. E. L. Green), pp. 351-372. McGraw-Hill, New York.

SABIN, F. R. (1920). Studies on the origin of blood-vessels and of red blood corpuscles as seen in the living blastoderm of chicks during the second day of incubation. Contrib. Embryol. 9 , 215-262.

Samarut, J. and Gazzolo, L. (1982). Target cells infected by avian erythroblastosis virus differentiate and become transformed. Cell 28, 921-929.

Thompson, J. AND Gillespie, D. (1987). Molecular hybridization with RNA probes in concentrated solutions of guanidine thiocyanate. Anal. Biochem. 163, 281-291.

Till, J. E. AND MCCulLoCH, E. A. (1980). Hemopoietic stem cell differentiation. Biochim. biophys. Acta. 605, 431-459.

Traganos, F., Darzynkiemicz, Z., Sharpless, T. K. and
Melamed, M. R. (1979). Erythroid differentiation of Friend leukemia cells as studied by acridine orange staining and flow cytometry. J. Histochem. Cytochem. 27, 382-389.

Virtanen, I., Kurkinen, M. AND Lehto, V.-P. (1979). Nucleusanchoring cytoskeleton in chicken red blood cells. Cell Biol. Int. Rep. 3, 157-162.

WoOd, L., Theriault, N. AND Vogeli, G. (1989). Vimentin cDNA clones covering the complete intermediate-filament protein are found in an EHS tumor cDNA library. Gene 76, 171-175.

Zinn, K., DiMaio, D. ANd Maniatis, T. (1983). Identification of two distinct regulatory regions adjacent to the human binterferon gene. Cell 34, 865-879. 